Physical Sciences | Michael K. Denk

\title{
Classic biomolecules continue to surprise with unexpected reactivity
} and folates were identified

by early biochemists for their hydrogen in fermentation and hydrogen transfer and are today Che Chemistry at the University f Guelph, PhD candidate Nicholas Milutinovic and PhD candidate Katherine Marczenko have been performing research which has shown that these old biomolecules can still turn up new surprises. These molecules ay have great potential damaging halogenated organic molecules like the notorious pesticide at the centre of the early environmental movem DDT.

I's hard to say which Alfred Nobel is more famous for: creating his eponymous Prizes, or inventing dynamite. Nobel's objective with dynamite, at least originally, was to make safer the fiercely dangerous mining explosive inventions would also enable a vast number of deaths during war, and its hard to ignore that arms sales earned $h$ much of his fortune.

When Alfred's brother Ludvig died in 1888 , the story goes that a French newspaper published an obituary for Alfred in error, titled "Le marchand de mort est mort": "The merchant of death is dead", an event often cited as being the reason he dedicated almost all of $h$ wealth to the prestigious Nobel Prizes. In 1948, the Nobel Prize for Physiology or Medicine was awarded to Swiss chemis Paul Hermann Maller for his dscovery This insecticidal properties of DDT. especilly in the USA wher itwase,

a commercial and household pesticide. DDT was notably used during and immediately
after World War II to kill the insects which transmit malaria and typhus.

Over time, research emerged sugges that the pesticide posed a risk to health, and after decades campaigning initiated primarily by Rache use in the United States i 1972 . L Nobel's dynamite, Müller's Nobetwinning DDT saved and still does save
Ives - possibly even millions - but with

'FOREVER CHEMICALS'

Despite heavy regulation, DDT and its metabolites persist in the environmen DDT is a halogenated organic molecule long time to be broken down in the environment DDT is either broken down by light or is ingested by organisms, where it can bioaccumulate, most notably by birds at the top of the food chain. Through eggshell thinning, DDT is thought to be responsible for the decline of bald eagles, brown pelicans, peregrin falcons, and ospreys.

\section{Removing these molecules from the} environment remains a challenge. One of the problems is that, whilst the metabolites or DDT are well-documented, me biological processes by which these understood even after decades of research interest.

\section{DEHALOGENATION}

The first step of metabolising DDT 'dehalogenation', a process found in all organisms studied to date. This process was first identified in yeast in 1963, and was later confirmed in mammals. In the chemistry lab, the same transformations are performed using organometallic reagents, the most commonly used being tin hydrides and hydrosilanes. These molecules are hydride donors, meaning they provide a source of negative

Dr Michael Denk, Professor of Chemistry at the University of Guelph, and PhD candidate Nicholas Milutinovic, began studying the interaction between halocarons and biomolecules when called imidazolidines were unstable in the commonly-used halogenated solvent chloroform.

Closer examination showed that imidazolidines act as hydride donors, converting halocarbons to hydrocarbons. Continuing their investigations, Denk and Milutinovic confirmed that this new type of reaction is actually very general, able to dehalogenate a wide variety of halogenated hydrocarbons, including those with environmental and medicinal relevance. The dehalogenation is particularly effective for brominated halocarbons, including the inert fla proceeding win mintes at room temperature.

The medicinal anaesthetic halothane (2-bromo-2-chloro-1,1,1-trifluoroethane), which has fluorine, chlorine, and bromine in its structure had its bromine atom ed by the imidazolines.

\section{FOLATES - THE MISSING LINK IN} HALOCARBON METABOLISM?

At the very least, this unusual reactivity puts imidazolines forward as a candidate for the replacement of the toxic tin hydrides commonly used in selective debromination and dechlorination of molecules. But what makes this discovery really remarkable is that a a so the of 5,10-methylenetetrahydrofolte (MTHF). MTHF is a highly-studied and biologically ubiquitous hydride donating biomolecule, involved in DNA methylation and the synthesis of DNA bases and amino acids in cells.

The discovery that imidazolines are ble to perform these dehalogenation reactions, even in the presence of air or moisture, means these reactions are feasible in cellular conditions - as is the possibility that MTHF is responsible for the dehalogenation steps in metabolic degradation of halogenated organic molecules. Despite their simple chem structure, inidazolidnes are rare in to date participate in very significant

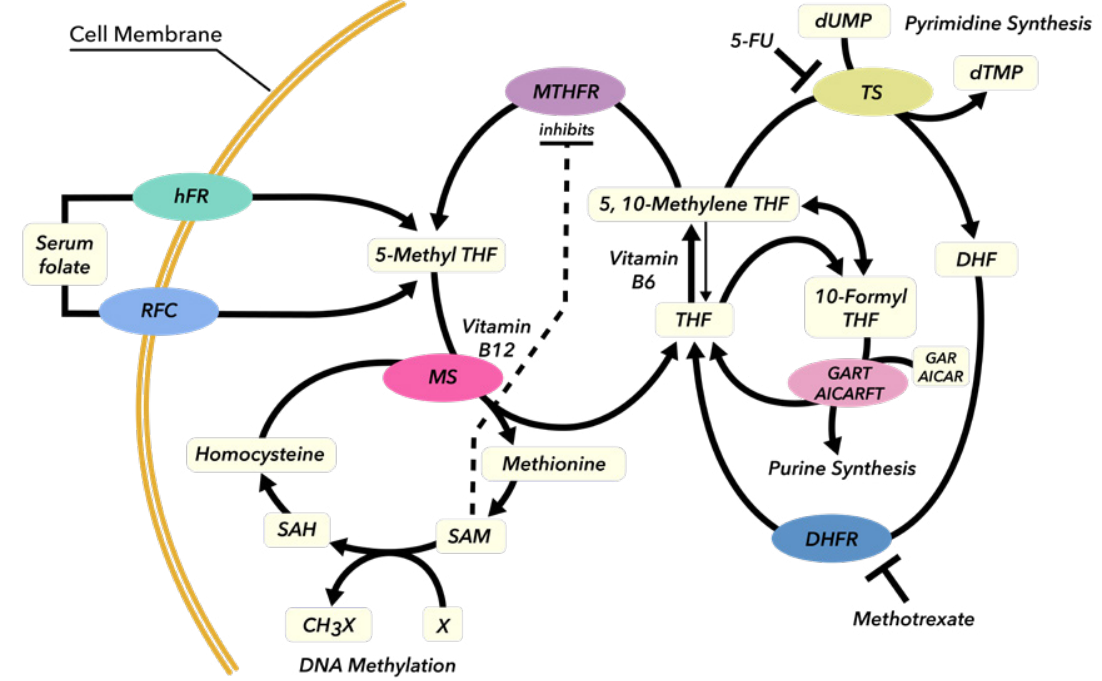

functions. Another imidazolidine biomolecule, methenyl-H4MPT, found in species of methanogenic archaea, is for splitting molecular hydrogen - one of the strongest chemical bonds known

This fascinating reactivity has led Denk's group to collaborate with McMaster University biochemist and hydrogenase expert Athanasios Paschos to unlock th full breadth of folate chemistry hidden cells and test tubes alike. Prof Paschos

be responsible for dehalogenating DDT cytochrome P450 enzymes, nicotinamid denine dinucleotide phosphate and flavin adenine dinucleotide PADH) have all been implicated over the past decades.

\section{EFFECT OF FOLATE
MODELS ON DDT}

Despite its ubiquity as a biological hydride donor, MTHF has not previously

Whilst the metabolites of DDT are welldocumented, the biochemical processes by which these metabolites are formed are still poorly understood.

is comparing the in vivo activity of his to that of the in vitro folate models in order to develop microbial approaches to halocarbon remediation and

hydrogen generation.

Despite the volume of environmenta and toxicological research done as a result of the concerns around DDT, little is known about how it is metabolised. Many biomolecules have been postulated to

dehalogenation metabolism of DDT, or any other halogenated organic that imidazolidines are capable of dehalogenating many other halogenated hydrocarbons, Denk and Milutinovic began investigating the effect on DDT of imidazolidines as model compounds for MTHF.

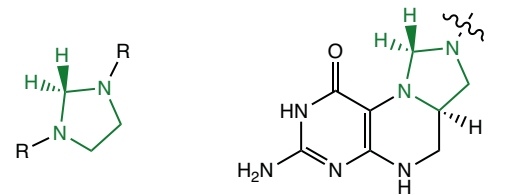
was investigated using two protocol

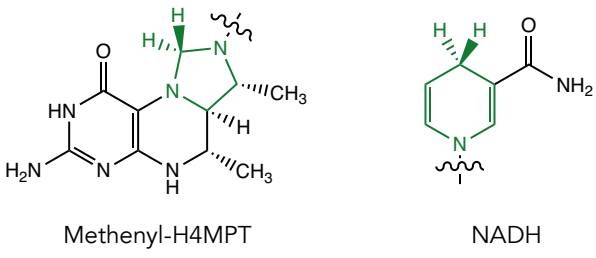




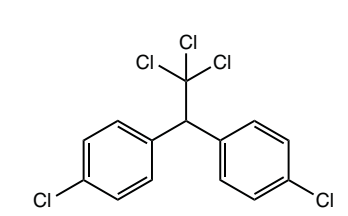

DDT
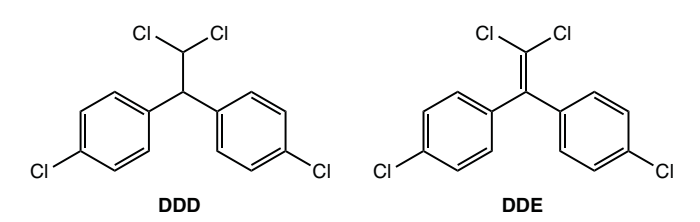

(1)<smiles></smiles>

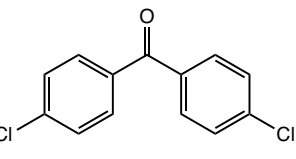

Metabolites of DDT. The first step of metabolising DDT is 'dehalogenation'.

- one aerobic and one anaerobic. The same metabolites as seen in vivo und the same conditions were produced in these reactions, using multiple MTHF model compounds. Particularly foresting examples include the which has up unil now to be produced by cytochrome P450. Denk and Milutinovic's observations show that DBP can be obtained from MTHF models under aerobic conditions without cytochrome P450.

These metabolic degradation products have been known for over 50 years, but the way they are formed in vivo has remained a mystery. Denk and Milutinovic's findings are compelling evidence that MTHF is the biological reducing agent involved in the metabolic degradation of DDT.

METABOLIC DISRUPTION This newly identified role of MTHF in the breakdown of DDT and other halocarbons has interesting implicat When MTH breaks down DDT in cells, it is itself depleted. That is good reason to believe that DDT is therefore involving MTHF which may explain why DDT is so damaing to insects mammalian cells.

Denk and Milutinovic's most recent research has expanded to biologica hydride donors beyond MTHF. Of particular interest is NADH, the crucia biological agent converting sugar into alcohol and the first biological hydrid transfer agent identified as such. Their research shows that NADH is also capable of generating most of the DDT intro, and so its role

\section{Denk and Milutinovic's findlings are} compelling evidence that MTHF is the biological agent involved in the metabolism of DDT and halocarbons in general.
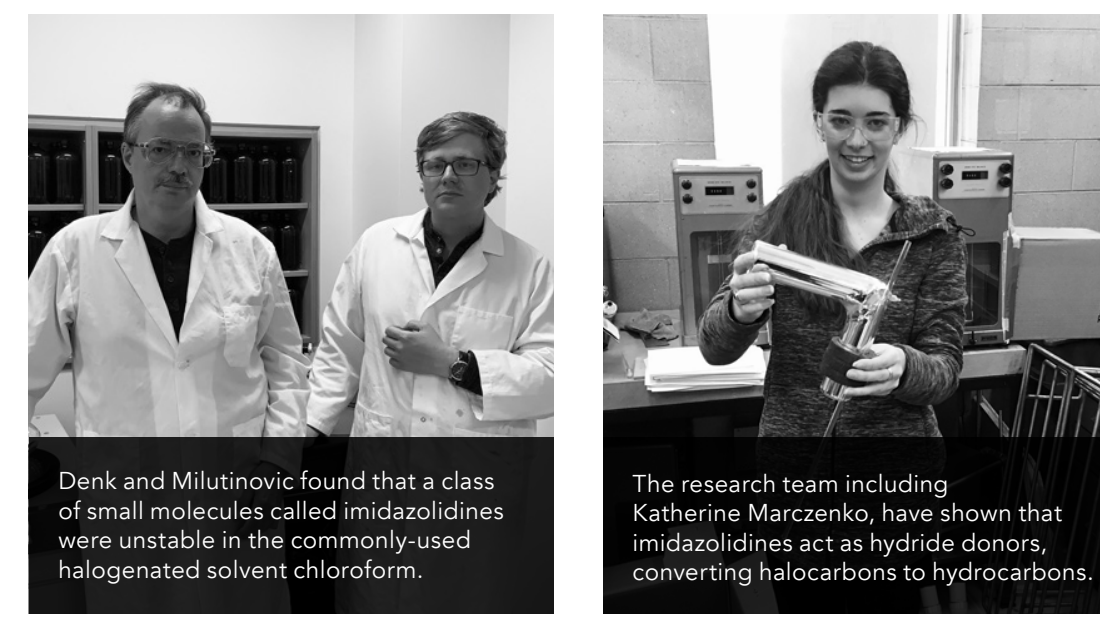

in the metabolising of DDT cannot be ruled out. In addition to carrying out uses high precision quantum chemical calculations to predict the outcomes of the chemical reactions. The calculations reveal that the NADH carbon-hydrogen bond energy - an important factor in the way a hydride donor interacts with other molecules - is significantly lower than that of MTHF. The good news provided by the calculations is that a organohalides - fluorides, chlorides, bromides, and iodides - can in principle be converted to hydrocarbons; the bad news is that some halocarbons are reduced only very slowly. Speeding up these tardy reactions by catalysts is the Denk group.

\section{FUTURE APPLICATION OF FOLATES}

Halogenated hydrocarbons are toxic to

the results from Denk's deplete folate levels in the body Low

folate levels are known to be a significant risk factor for cancer and birth defects. This has led Denk and Milutinovic to team up with medical researchers at Penn State University to explore how this information can be used to benefit human health. Penn State physician Robert Lennon is currently evaluating MTHF model compounds as topical reducing agents, to combat oxidative stress caused by reactive oxygen species generated development of skin cancers.

Evidence that DDT is broken down by olates also has promising implication environment The MTHF models are readily accessible and are capable of degrading a wide variety of halogenated compounds, in the presence of air and water, unlike many dehalogenation approaches - this gives them enormous potential to be used in the remediation of DDT waste sites.

The straighttorward synthesis of the folate molecules studied allows the production of tailor-made analogues. One of the most promising candidates for remediation is a polymeric version, which and is es cap re remediation sites nd is even capable of capturing heavy metals and precious metals.

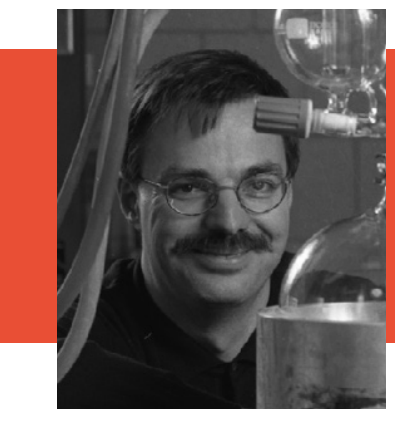

\section{Behind the Research}

\section{Dr Michael K. Denk}

E: mdenk@uoguelph.ca E: michaeldenk@me.com T: +1(647) 9199365

W: http://www.chemistry.uoguelph.ca/cgi-bin/uchem.exe?ac=v_page\&pa=W8F8OY

Research Objectives

Michael Denk's research explores folate intermediates, hydrogenases models, and hydride transfer reactions under biological conditions.

Detail

Michael Denk, University of Guelph

Sone Rd. East

Guelph, N1G 2W1. Canad.

Bio

Michael Denk was born in 1961 in Munich, Germany. He obtained his MSc from the Ludwig Maximilian University

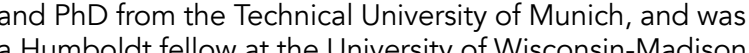
where he synthesised the first stable silylene moleculen He has conducted a scientific expedition to India and has travelled to Tibet, China, India, Japan, North Yemen, Sudan, . classical music, archaeology, and languages.

Funding

Province of Ontario (fellowships for NSM)

- Guelph Institute for Environmental Research (GIER) grant

- Prof Mario Monteiro contributed funding to parts of this research through an NSERC award

Collaborators

Nicholas Milutinovic

- Robert Lennon

- Katherine Marczenko

\section{References}

Denk, M.K., Milutinovic, N.S., Marczenko, K.M., Sadowsk reduction of halocarbons by folate model compo Chem. Sci. 8. 1883-1887. https://doi.org/10.1039/

- C6SC04314C of the DDT metabolite spectrum under biomimetic conditions. Chemosphere 191, 408-411. doi:10.1016/i

$\frac{\text { chemosphere.2017.10.055 }}{\text { Denk, M.K., Milutinovic, N.S., Dereviankin, M.Y. (2019) }}$ Rodels on $\mathrm{NADH}$ Chems to hydrocarbons by NADH doi:10.1016/i.chemosphere.2019.05.169 Lough, J. (2001) Oxidation, hydrogenation and hydrolysis 618, P242-253 doi101016/S0022-328x/a0) Ch551-9.

\section{Personal Response}

\section{What would be the next steps in your remediation}

II For in situ remediation, we need a material that can stay in the ground for prolonged periods of time withou you will. Nicholas has created a polymer that does exacty that. An equally important goal is to better understand how we can accelerate the reactions by finding

Reduction is a very general process. Do you forese Reyond halocarbons?

II Mining runoff from slags and process residues contains traces of carcinogenic metal ions like arsenic, chromium, and lead. These ions can be reduced to free
metals for recovery, and our MTHF models offer a new water-compatible approach.

II A second topic that is still in the discussion stage is an important class of chemotherapeutics for cancer The general idea is to protect healthy cells with our hydride transfer agents without impairing the toxicity of the cancer drug to cancer cells.

Our colleague Robert Lennon, MD, at Penn State compounds as topical agents to fight oxidative stress from reactive oxygen species. These are commonly loxins. This approach may reduce carcinogenesis and also decrease the effects of ageing on the skin.

Discussions with our colleague Aiguo Ren from Peking the potential use of MTHF and related molecules to lessen the risk of birth defects. Prof Ren has presented strong evidence linking halocarbons and DDT in particular to neural tube defects. As there is no method to remove the highly lipophilic halocarbon insecticides
from living organisms, fighting their detrimental effects at the molecular level seems promising 